\title{
A Vehicle Detection Approach from Satellite Imagery Using Antibody Networks
}

\author{
Hong Zheng ${ }^{1}$ ， Xuemin Hu \\ Joint Lab for Intelligent Computing and Intelligent Systems, School of Electronic Information \\ Wuhan University, 129 Luoyun Road, Wuhan, Hubei 430079, P.R. China
}

\begin{abstract}
This paper presents a novel vehicle detection approach based on artificial immune principle. The approach is to build an artificial immune system for vehicle detection from high resolution satellite imagery, where vehicles mimic dangerous antigens and vehicle detection templates mimic antibodies. The artificial immune system uses the antibody network concept inspired from the immune system to learn a set of templates called antibodies for vehicle detection. The details of proposed approaches are described in this paper, and experiments on real satellite images are also given to show the feasibility and performance of proposed method.
\end{abstract}

Keywords: Antibody network; Vehicle detection; Satellite imagery

\section{Introduction}

With the development of traffic there is high demand in traffic monitoring of urban areas. Currently the traffic monitoring is implemented by a lot of ground sensors like induction loops, bridge sensors and stationary cameras. However, these sensors partially acquire the traffic flow on main roads. The traffic on smaller roads - which represent the main part of urban ${ }^{1}$ road networks - is rarely collected. Furthermore, information about on-road parked vehicle is not collected. Hence, area-wide images of the entire road network are required to complement these selectively acquired data. Since the launch of new optical satellite systems like IKONOS and QuickBird, this kind of imagery is available with 0.6-1.0 meter resolution. Vehicles can be observed clearly on these high resolution satellite images. Thus new applications like vehicle detection and traffic monitoring are raising up. This paper intends to study the vehicle extraction issue from high resolution satellite images.

Some vehicle detection methods have been studied using aerial imagery [1-4]. In the existing

${ }^{1}$ Corresponding author.

Email address: hzheng@inass.org methods, two vehicle models are used. They are explicit model and appearance-based implicit model. They describe a vehicle as a box or wire-frame representation. Detection is carried out by matching the model "top-down" to the image or grouping extracted image features "bottom-up" to create structures similar to the model. However, for 0.6-1.0 meter resolution satellite images, vehicle image detail is too poor to detect a vehicle by model approaches. So far, few research on vehicle detection from high-resolution satellite imagery has been reported [5-7]. Among these researches, the method based on morphology share-weight neural network (MSNN) is the best. But it depends on the training of vehicle samples and non-vehicle samples collected from road surfaces. Since road surface in satellite images is usually too complex to collect various non-vehicle samples, it sometimes lead to missing detection and false detection. Thus, it is necessary to develop new approaches to detect vehicles from high resolution satellite imagery.

The immune system is one of the highly evolved biological information processing systems and is capable to learn and memorize. In recent years, artificial immune systems have been successfully applied to solve many engineering problems [8-10]. In our study, we attempt to apply them in target 


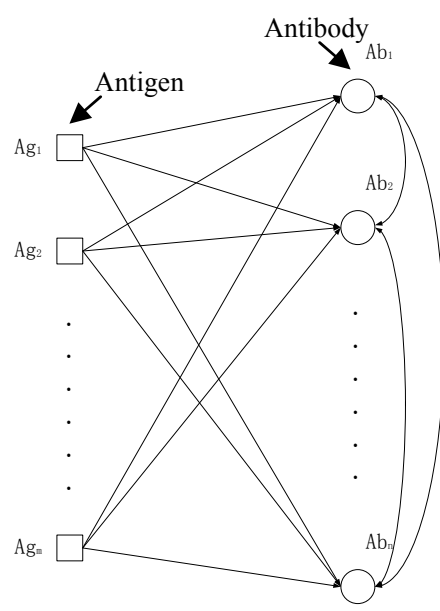

(a)

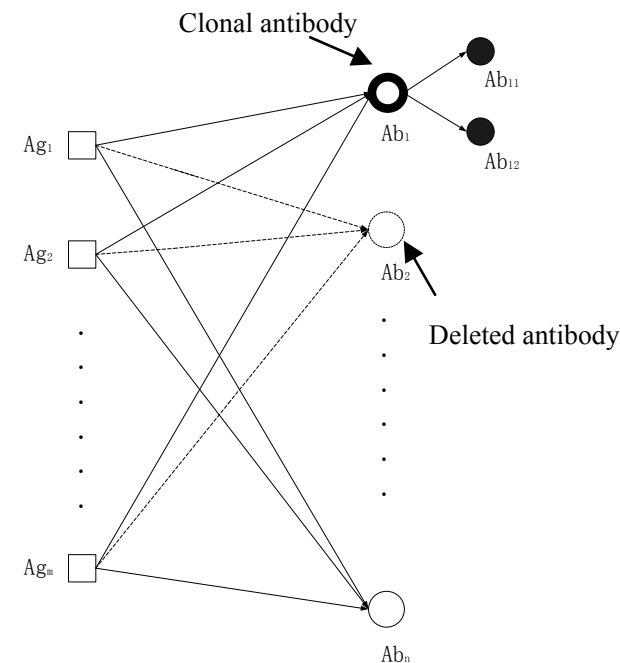

(b)

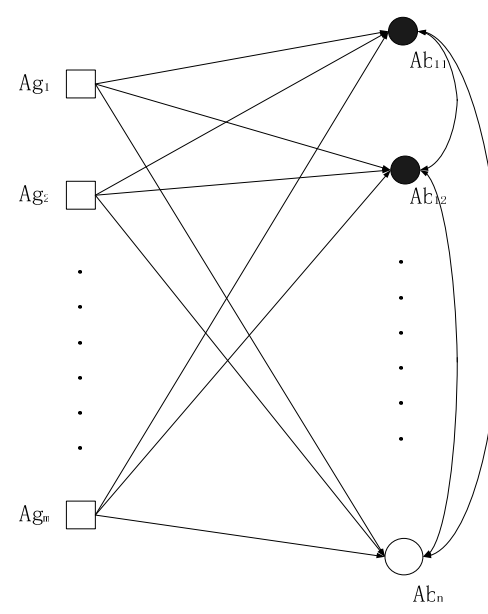

Figure 1. (a) An original ABNET. (b) ABNET growing and pruning process. (c) A new ABNET.

recognition. Interested targets are regarded as foreign antigens, and detection templates are regarded as antibodies. Complementary template matching is considered to be exactly the same as the binding of the paratope and epitope. In order to obtain these antibodies, we propose an antibody network to acquire vehicle detection antibodies only using vehicle samples.

In this paper, we concentrate the vehicle detection on roads and parking lots, which can be manually extracted in advance. In order to collect vehicle contour samples, a preprocessing algorithm based on bilateral filtering and morphology is developed. The algorithm implements bilateral filtering and morphology operations on images to remove noises and enhance vehicle features. Based on preprocessed images, some vehicle samples are collected as the training samples for antibody learning. A set of template antibodies are learned using proposed antibody network and tested on real road segments and parking lots, which are collected from Quickbird satellite images. Experimental results and comparison results with MSNN based method are also discussed in this paper.

The paper is organized as follows. In Section 2, the details of our vehicle detection approach are described. In Section 3, experimental results are given and conclusions are provided in Section 4.

\section{Immune vehicle detection system based on antibody network}

\subsection{Overview of Antibody Networks (ABNET)}

ABNET is a Boolean competitive neural network model, based upon properties of the clonal selection paradigm of the immune system. The antibody repertoire is modeled using a connectionist approach, with an antibody network being generated, where the connection strengths of the network represent the molecules, and the recognition is performed in a systemic level, rather than by single individuals [11].

The main features of the antibody network are as follows [11]:

- growing architecture, based on the clonal selection principle;

- network pruning, representing the death of non-stimulated cells (apoptosis);

- boolean connection strengths; and

- competitive network, with unsupervised learning based on a mutation mechanism.

ABNET consists of two important processes: network growing and pruning. The growing process mimics the selection and reproduction of the most stimulated cell, according to the clonal selection principle, and the pruning process is to delete an immune cell from network, whose concentration level equals to zero longer than a specified length of time. Figure1 illustrates an ABNET and its two processes. In Figure 1, an antibody interact with not only antigens but also other antibodies, and the weight value between an antigen and an antibody means whether the antibody is able to recognize the antigen. If the weight value is 1 , it means that the 
Table 1. Definition of immunological terms for vehicle detection

\begin{tabular}{|c|c|}
\hline Biology immune system & Vehicle detection system \\
\hline Antigen & Pre-processed vehicle objects on road surfaces \\
\hline Antibody & Detection templates \\
\hline Cell mutation & Inverting elements in a template \\
\hline ABNET growing & Adding vehicle samples into antibody database \\
\hline ABNET pruning & Delete bad antibodies from antibody database \\
\hline Innate immune layer & Image pre-processing \\
\hline Danger cognition & Vehicle feature extraction \\
\hline Antibody concentration & Recognition rate of a template \\
\hline Affinity & Correlation coefficient of a template and a detected object \\
\hline
\end{tabular}

antibody is able to recognize the antigen, otherwise it means that the antibody is not able to recognize the antigen.

ABNET has been used to solve some problems in engineering and pattern recognition and reached satisfactory results [8-10]. In fact, vehicle detection is of pattern recognition issue, therefore it is reasonable to apply ABNET in vehicle detection. As a result, our study employs ABNET to learn antibodies for vehicle detection.

\subsection{Definition of immunological terms for vehicle detection}

To build an artificial immune system for vehicle detection, some concepts corresponding to immunology terms should be defined firstly. These concepts are listed in Table 1.

According to immune principle, there is an innate immune layer in a immune system. For the vehicle detection issue, the innate immune layer is called as preprocessing layer. The preprocessing starts with bilateral filtering followed by a morphology transform. Bilateral filtering is a non-linear filtering technique introduced by Tomasi et al. It extends the concept of Gaussian smoothing by weighting the filter coefficients with their corresponding relative pixel intensities. Pixels that are very different in intensity from the central pixel are weighted less even though they may be in close proximity to the central pixel. This is effectively a convolution with a non-linear Gaussian filter, with weights based on pixel intensities. This is applied as two Gaussian filters at a localized pixel neighborhood, one in the spatial domain, named the domain filter, and one in the intensity domain, named the range filter [12]. In our study, the bilateral filtering technique is employed to remove noise on the road surface such as road marks and oil dust. The output of the bilateral filtering can be denoted by [12]: $k(x)=\int_{-\infty}^{+\infty} \int_{-\infty}^{+\infty} c(\varepsilon, x) s(f(\epsilon), f(x)) d \varepsilon d x$

Where $c(x)=e^{-0.5\left(\frac{d(\varepsilon, x)}{\sigma_{d}}\right)^{2}}$

$$
s(f(\varepsilon), f(x))=e
$$

$c(\varepsilon, x)$ is closeness function between central pixel $\mathrm{x}$ and its nearby pixel $\varepsilon$;

$s(f(\varepsilon), f(x))$ is the similarity function of central pixel $\mathrm{x}$ and its nearby pixel $\varepsilon$;

$d(\varepsilon, x)$ is the Euclidean distance between central pixel $\mathrm{x}$ and its nearby pixel $\varepsilon$;

$\delta(f(\varepsilon), f(x))$ is the absolute intensity difference between central pixel $\mathrm{x}$ and its neighborhood pixel $\varepsilon$

$\sigma_{r}$ and $\sigma_{d}$ are Gaussian gray standard errors.

The used morphology transform is to enhance vehicle contour features. It is defined by

$$
G(f)=f \oplus g-f
$$

Where $g$ is a 3 by 3 structuring element, $f$ is a gray image, $\oplus$ means a dilate operation (for details, please refer to reference [13]).

Figure 2 shows an original image and its bilateral filtering and morphology processing results. It can be clearly seen that most road marks are removed and all vehicle bodies or contours are enhanced. These enhanced features can be used to discriminate vehicle targets and non-vehicle targets. Figure 3 shows some vehicle examples collected from preprocessed image.

Affinity is a kind of matching index, which is inspired from image correlation concept. It is defined by

$$
R=\frac{\sum_{x=0}^{L-1} \sum_{y=0}^{K-1}(w(x, y)-\bar{w})(f(x, y)-\bar{f})}{\sqrt{\sum_{x=0}^{L-1} \sum_{y=0}^{K-1}(w(x, y)-\bar{w})^{2}} \sqrt{\sum_{x=0}^{L-1} \sum_{y=0}^{K-1}(f(x, y)-\bar{f})^{2}}}
$$

Where $w(x, y)$ is the template antibody image with 
size $K X L, f(x, y)$ is the antigen image with size $K X$ $L, \bar{w}$ is the average intensity value of the pixels in template antibody image $\mathrm{w}, \bar{f}$ is the average intensity value of the pixels in template antigen image $f$. The greater the value of $R$, the higher the antibody's affinity.

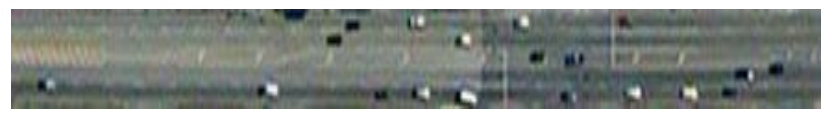

(a) An original image

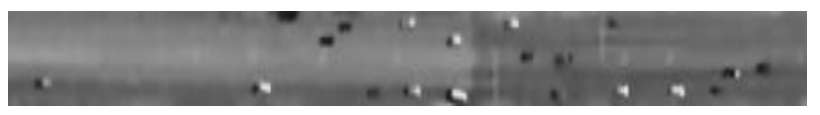

(b) The bilateral filtering result

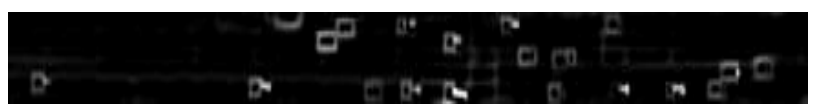

(c) The morphology transform result.

Figure 2. An original image and its bilateral filtering and morphology preprocessing results.

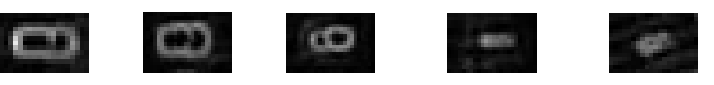

Figure 3. Vehicle examples

\subsection{Learning antibodies for vehicle detection}

For antibody learning, we build a vehicle samples database firstly. In the database, all samples are collected from preprocessed images using same sampling window. We randomly select $\mathrm{N}$ vehicle samples from the database as the initial antibody population, the rest samples are regarded as training sets. According to the antibody network theory, antibodies interact each other and with the environment (antigens). The interaction property leads to the establishment of a network. When an antibody recognizes an epitope or an idiotope, it can respond either positively or negatively to this recognition signal. A positive response would result in antibody activation, antibody proliferation and antibody secretion, while a negative response would lead to tolerance and suppression. According to these properties, we develop an antibody network for vehicle detection. A set of rules are proposed for antibody selection and updating in the immune network. These rules are listed as follows.

\section{Rule 1: Network pruning rule}

1) Eliminate one antibody if the maximum affinity of the antibody to all antigens (vehicle samples) is under the given affinity threshold.

2) Eliminate one antibody if the antibody's similarity to some other antibody is over the given similarity threshold and its antibody concentration (recognition rate) is less than that of the similar antibody.

\section{Rule 2: Network growing rule}

1) Add a vehicle sample from training sets into the antibody population as a new antibody if the affinity of the vehicle sample to any antibody is under the given affinity threshold.

2) If all vehicle samples can be recognized by current antibody population, $\mathrm{K}$ antibodies are generated randomly and added into current antibody population as new antibodies.

\section{Rule 3: Mutation rule}

Randomly select one antibody from deleted antibodies, and randomly invert one or more element in the antibody according to following equation:

$$
f=255-g
$$

Where $f$ is the mutation result, $g$ is the gray value of selected element in selected antibody.

Based on above rules, the antibody learning procedure in the immune network is described as follows:

Step 1: Randomly select $\mathrm{N}$ vehicle samples from the database as the initial antibody population, and setup affinity threshold and similarity threshold;

Step 2: Evaluate the affinity of each antibody in the population with Eq. (5);

Step 3: Implement network pruning by eliminating antibodies according to Rule 1;

Step 4: Implement network growing by updating antibody population according to Rule 2;

Step 5: Implement mutation operation according to Rule 3;

Step 6: Repeat Steps 2-5 until none of antibodies is eliminated and none of new antibodies is added in continued 3 iterations.

Step 6: Output final antibody population for vehicle detection.

\subsection{Vehicle detection procedure}

After learning the antibody population, the learned antibody population can be used to detect vehicles in the imagery.

Firstly, according to Eq.(1) and Eq.(3), implement bilateral filtering and morphology transform on the original image. Secondly, calculate the maximum affinity among all template antibodies at each pixel point by Eq.(5). Thirdly, compare the maximum affinity value $R$ at every point with the given threshold. If the $\mathrm{R}$ is greater than the given 


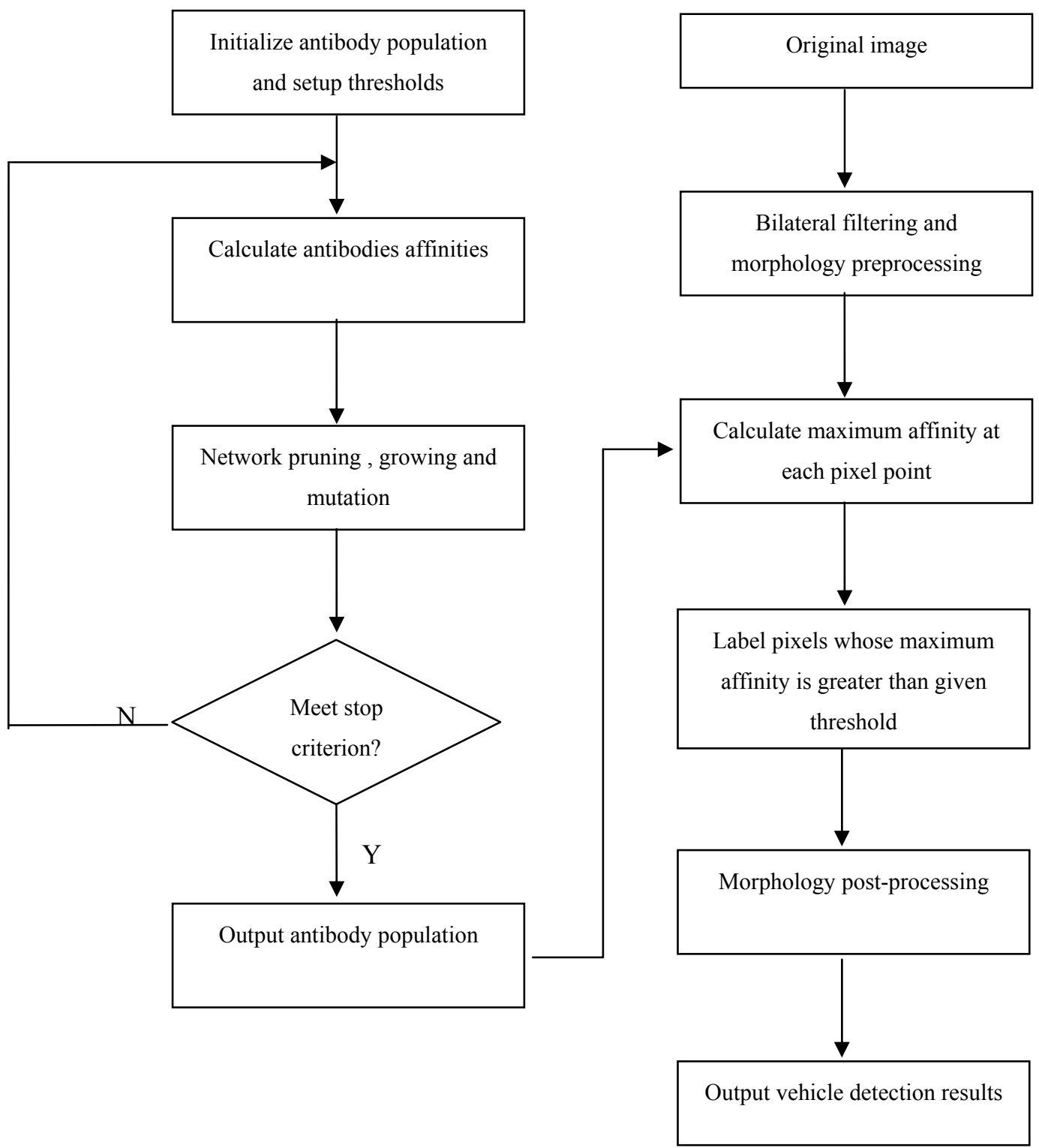

Figure 4. The flowchart for antibody learning and vehicle detection.

threshold, the point belongs to a vehicle target and is set to 255. Otherwise, it belongs to a non-vehicle target and is set to 0. Finally, a post-processing based on morphology dilation and erode operations is employed to merge neighborhood vehicle target pixels and locate the center of a vehicle.

Figure 4 shows the flowchart of the antibody learning and vehicle detection based on learned antibodies.

\section{Experimental results}

QuickBird panchromatic data sets used in our study were collected from Space Imaging Inc. web site. The data sets contain different city scenes. A total of 12 road segments and 4 parking lots segments containing over 1000 vehicles were collected. Most vehicles in the images are around 5 to 10 pixels in length and around 3 to 5 pixels in width. Since the vehicles are represented by a few pixels, their detection is very sensitive to the surrounding context. Accordingly, the sample database consist of 100 vehicle samples from variety of road conditions, such as road intersections, curved and straight roads, roads with lane markings, road surface discontinuity, pavement material changes, shadows cast on the roads from trees, etc. These represent most of the typical and difficult situations for vehicle detection. 
Table 2 Vehicle detection comparison results of MSNN based method and proposed method

\begin{tabular}{|c|c|c|c|c|c|}
\hline Site & $\begin{array}{c}\text { No. of } \\
\text { vehicles }\end{array}$ & $\begin{array}{c}\text { No. of detected } \\
\text { vehicles }\end{array}$ & $\begin{array}{c}\text { No. of missing } \\
\text { vehicles }\end{array}$ & $\begin{array}{c}\text { No. of false } \\
\text { alarm }\end{array}$ & Detection rate $\%$ \\
\hline Road1 & 6 & $6 / 6$ & $0 / 0$ & $0 / 0$ & $100 / 100$ \\
\hline Road2 & 8 & $7 / 8$ & $1 / 0$ & $0 / 0$ & $87.5 / 100$ \\
\hline Road3 & 11 & $9 / 10$ & $2 / 1$ & $2 / 1$ & $81.8 / 90.9$ \\
\hline Road4 & 15 & $13 / 15$ & $2 / 0$ & $1 / 0$ & $86.6 / 100$ \\
\hline Road5 & 20 & $16 / 17$ & $4 / 3$ & $2 / 2$ & $80 / 85$ \\
\hline Road6 & 18 & $15 / 17$ & $3 / 1$ & $1 / 0$ & $83.3 / 94.4$ \\
\hline Road7 & 28 & $23 / 24$ & $5 / 4$ & $5 / 2$ & $82.1 / 85.7$ \\
\hline Road8 & 54 & $41 / 41$ & $13 / 13$ & $6 / 4$ & $75.9 / 75.9$ \\
\hline Road9 & 82 & $58 / 65$ & $24 / 17$ & $10 / 5$ & $70.7 / 79.2$ \\
\hline Road10 & 133 & $90 / 100$ & $43 / 33$ & $14 / 7$ & $67.6 / 75.1$ \\
\hline Road11 & 210 & $155 / 170$ & $55 / 40$ & $21 / 10$ & $73.8 / 80.9$ \\
\hline Road12 & 304 & $220 / 240$ & $84 / 60$ & $35 / 18$ & $72.3 / 78.9$ \\
\hline Parking1 & 7 & $7 / 7$ & $0 / 0$ & $0 / 0$ & $100 / 100$ \\
\hline Parking2 & 15 & $11 / 11$ & $4 / 4$ & $0 / 0$ & $73.3 / 73.3$ \\
\hline Parking3 & 46 & $28 / 30$ & $18 / 16$ & $4 / 5$ & $60.8 / 65.2$ \\
\hline Parking4 & 170 & $112 / 102$ & $58 / 68$ & $10 / 10$ & $65.8 / 60$ \\
\hline
\end{tabular}

For each selected road segment image or parking lot image, roads and parking lots were extracted manually in advance and vehicle detection was performed only on the extracted road surfaces. To build the vehicle example database, a human expert manually delineated the rectangular outer boundaries of vehicles in the imagery. A total of 100 vehicles delineated in this manner from 5 road segments. An image region with size $6 \times 6 \mathrm{~m}$ can cover most vehicles in the imagery. Hence, sub-images with size $10 \times 10$ pixels centered at vehicle centroids were built into the vehicle example database.

After building sample databases, vehicle samples were used to learn antibodies and validate the vehicle detection approach. We randomly select 30 vehicle samples from the database as the initial antibody population, the rest samples are regarded as training sets. The affinity threshold and similarity threshold are set to 0.6 and 0.7 respectively. According to learning algorithm in Section 2, we finally got an antibody population including 18 template antibodies.
Taking vehicle orientations into account, each template antibody was rotated every $45^{\circ}$ and the resulting template antibodies were also collected in the antibody population. As a result, the antibody population consisted of $18 \times 4=72$ template antibodies.

After learning, the antibody population was tested on 12 road segments and 4 parking lots. The detection statistical results are shown in Tables 2. In order to evaluate the performance of proposed method, MSNN based method is trained using same vehicle training samples, and tests on the same road segments and parking lots. The comparison results with proposed method are also listed in Table 1, where numerator means the results using MSNN based method and denominator means the results using proposed method. The detection rate equals to that of number of detected vehicles over the total number of vehicles. Figure 5 shows some images of vehicle detection results, where red regions represent detected vehicles.

From Table 1, it can bee seen that the detection rate of proposed method is from $75.1 \%$ to $100 \%$, 
and average detection rate is $87.1 \%$, while the detection rate of MSNN based method for road segments is from $67.6 \%$ to $100 \%$, and average detection rate is $80.1 \%$. The detection rates vary with the complexity of road surfaces, as well as the number of false alarm and missing detection. Generally, the missing rate and false alarm rate of MSNN based method are greater than that of proposed method. It is because MSNN based method depends on learning vehicle and non-vehicle samples, but in the experiments, only limited vehicle samples are learned and none of non-vehicle samples is involved.

The false alarms are due to vehicle-like "blobs" present in some of complex urban roads such as trees and lane markings (see Figure 5). Some of these "blobs" are very hard to distinguish from actual vehicles, even to a trained eye. Most missing

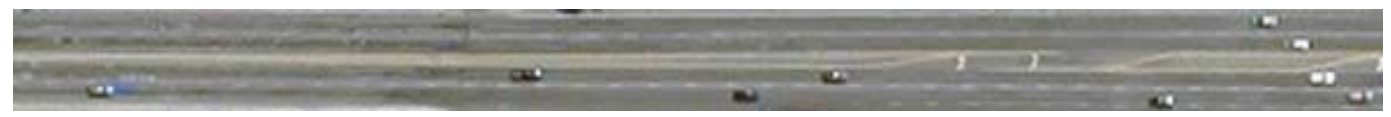

(a)

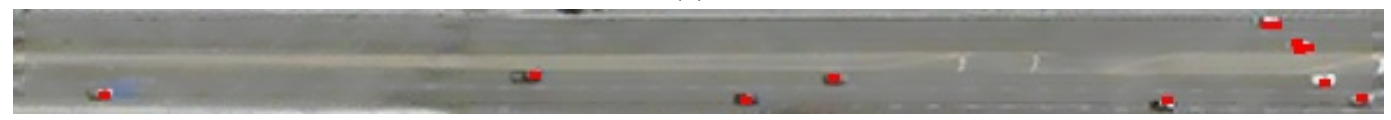

(b)

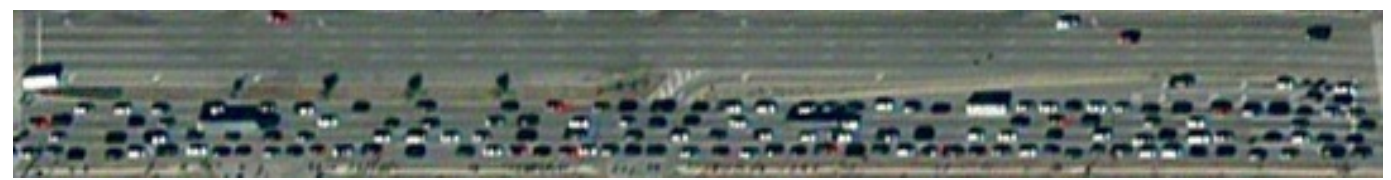

(c)

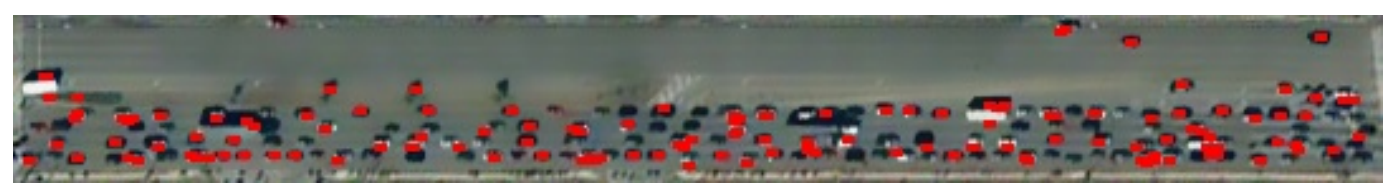

(d)

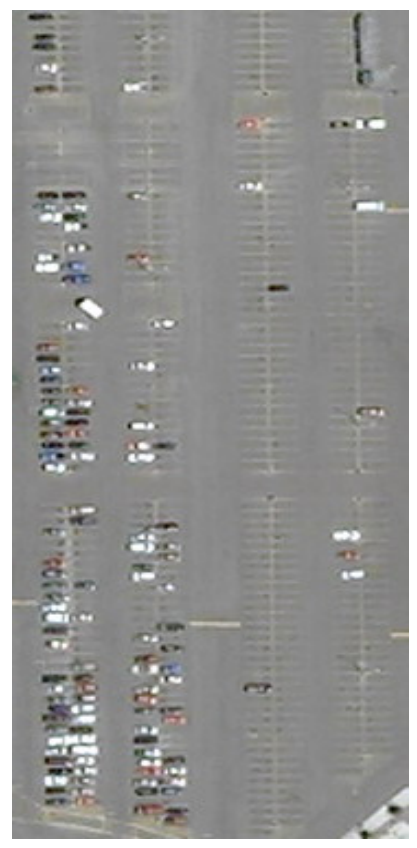

(e)

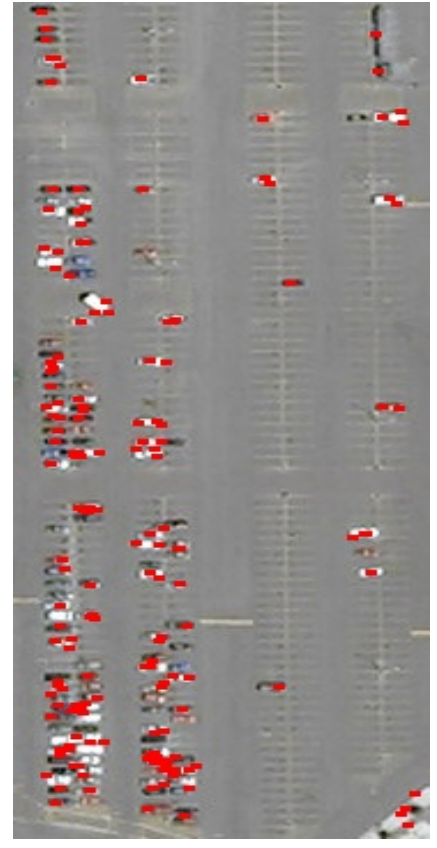

(f)

Figure 5. Vehicle detection results. (a)(c)(e) The sample images of road segments and parking lots. (b)(d)(f) The vehicle detection results for images shown in (a)(c)(e), where red regions represent detected vehicles. 
detections occur when the vehicles have a low contrast with the road surface or vehicles are too close. For the vehicle detection on parking lots, the performance of both methods is not good. It is because the vehicles are too close to separate due to the resolution limit. How to detect vehicles on parking lots is still an open issue. Comparing with other vehicle detection approaches [5,6], the proposed method directly detect vehicles on single satellite image, while Sharma's method needs a set of multi-temporal images at the same site to reach good detection performance [5]. A. Gerhardinger proposed the detection method based on Features Analyst Module for Arc/Gis [6]. The method was reported to obtain over $90 \%$ correct detection rate. However, some interactive workload are needed and cost is relatively expensive due to using commercial software. In addition, their methods were mainly tested on simple road scene, tests on heavy traffic roads and parking lots were not discussed in Sharma and Gerhardinger's study .

\section{Conclusions}

In this paper, we focus on the issue of vehicle detection from high resolution satellite imagery. We proposes an novel approach for vehicle detection from 0.6 meter resolution panchromatic QuickBird satellite imagery. The concept of antibody network learning was introduced in our approach and was found to have good vehicle detection performance. Further work would mainly solve vehicle detection problems on heavy traffic roads and parking lots.

\section{Acknowledgments}

This work was supported by the National Nature Science Foundation of China under Grant No. 40571102.

\section{References}

[1] R. Ruskone, L. Guigues, S. Airault, and O. Jamet, "Vehicle Detection on Aerial Images: A Structural Approach", In: Proceedings of International Conference On Pattern Recognition, Vienna, Austria, pp.900-904, 1996.

[2] T. Zhao and R. Nevatia, "Car Detection in Low Resolution Aerial Image", In: Proceedings of International Conference on Computer Vision, Vancouver, Canada, pp. 710-717, 2001.

[3] C. Schlosser, J. Reitberger, and S. Hinz, "Automatic Car Detection in High Resolution Urban Scenes Based on An Adaptive 3D-model", In: Proceedings of the 2nd GRSS/ISPRS Joint Workshop on Data
Fusion and Remote Sensing over Urban Area, Berlin, Germany, pp. 167-170, 2003.

[4] U. Stilla, E. Michaelsen, U. Soergel, S. Hinz, HJ. Ender, "Airborne Monitoring of Vehicle Activity in Urban Areas, In: International Archives of Photogrammetry and Remote Sensing, Vol.35, pp. 973-979, 2004.

[5] G. Sharma, Vehicle Detection and Classification in 1-m Resolution Imagery, Ohio State University, Master of Science thesis, 2002.

[6] A. Gerhardinger, D. Ehrlich, M. Pesaresi, "Vehicles Detection from Very High Resolution Satellite Imagery", In: International Archives of Photogrammetry and Remote Sensing, Vol. XXXVI, Part 3/W24, pp.83-88, 2005.

[7]Xiaoying Jin, Curt H. Davids, "Vehicle Detection from High Resolution Satellite Imagery Using Morphological Shared-weight Neural Networks", Image and Vision Computing, Vol.25, pp.1422-1431, 2007.

[8] D. Dasgupta (Ed.), Artificial Immune Systems and their Applications, Springer-Verlag, Berlin, 1999.

[9] L.N. De Castro, F.J. Von Zuben, "The Clonal Selection Algorithm with Engineering Applications", in: Proceedings of the Genetic and Evolutionary Computation Conference, pp. 36-37,2000.

[10] L.N. Castro, and J. Timmis, Artificial Immune Systems: A New Computational Intelligence Approach, Springer-Verlag London Berlin Heidelberg, 2002.

[11] C. Tomasi, R. Manduchi, "Bilateral Filtering for Gray and Color Images", In: Proceedings of the 6th International Conference on Computer Vision, Washington D C, IEEE Computer Society, pp.839-846,1998.

[12] J. Serra, Image Analysis and Mathematical Morphology, Vol.2, Academic Press, New York, N.Y ,1988. 